\title{
Wave mixing rise inferred from Lyapunov exponents
}

\author{
Alvaro Galan · Alejandro Orfila · Gonzalo \\ Simarro · Ismael Hernández-Carrasco .
}

Cristobal Lopez

Received: date / Accepted: date

\begin{abstract}
We study the horizontal surface mixing and the transport induced by waves in a coastal environment. A comparative study is addressed by computing the Lagrangian Coherent Structures, via Finite Size Lyapunov Exponents, that arise in two different numerical settings: with and without wave coupled to currents. In general,
\end{abstract}

A. Galan

E.T.S.I. Caminos, Canales y Puertos. Universidad de Castilla la Mancha, 13072 Ciudad Real, SPAIN. Email: alvaro.galan@uclm.es

A. Orfila

IMEDEA(CSIC-UIB), 07190 Esporles, SPAIN.

G. Simarro

Institut de Ciencies del Mar ICM (CSIC), 08003 Barcelona, SPAIN.

I. Hernández-Carrasco

IFISC (CSIC-UIB). 07122 Palma de Mallorca, SPAIN.

C. Lopez

IFISC (CSIC-UIB). 07122 Palma de Mallorca, SPAIN. 
we observe that mixing is increased in the area due to waves. Besides, the methodology presented here is tested by deploying a set of eight Lagrangian drifters at different locations. This dynamical approach is shown as a valuable tool to extract information about transport, mixing and residence embedded in the Eulerian time dependent velocity fields obtained from numerical models.

Keywords Lyapunov Exponents · Lagrangian Coherent Structures · Mixing

PACS $10.522 \cdot 10.531 \cdot 40.200$

\section{Introduction}

Transport, dispersion and mixing of coastal waters are of crucial interest due to the ecological and economical importance of these areas. Despite the increasing advance in the scientific description of the physical processes taking place in the coastal ocean, a high degree of uncertainty still remains when predicting the dynamical properties of the flow in coastal environments. On the one hand, coastal dynamics is influenced by deep water conditions over a complex topography and driven at the surface by highly variable (spatially and temporally) wind forcing. On the other hand, the effects of wind generated waves modify the current field by the excess of momentum flux induced by waves, which is usually accounted in coastal ocean models by the radiation stress concept [15]. Therefore, flows in coastal and shallow regions are the combination of currents with variations of hours to days, and wave oscillatory flows with periods of seconds to tens of seconds.

Transport and mixing properties of the ocean surface have been widely studied from a Lagrangian perspective, i.e., by following fluid particle trajectories $[5,10,16$, 
17]. One of the main objectives is the detection of the Lagrangian Coherent Structures (LCS) that organize the whole flow $[4,6,9,11,13,18]$, i.e., vortices, barriers, etc. This is performed measuring the local dispersion of transported particles, in particular, with the so-called Finite Size Lyapunov Exponents (FSLE) [2,3]. LCS are the ridges of the FSLE field, and these are calculated by measuring the time it takes for two trajectories, initially separated a finite distance, to reach a larger final fixed separation (see next Section). Besides their suitability for studying LCS and dispersion properties, FSLE also provide extra information on characteristics time-scales which are very important in coastal environments. LCS have been studied in coastal environments using the FSLE and the different, but analogous, tool of the Finite Time Lyapunov Exponents for instance in [7,19].

In this work we analyze the influence of waves on the modification of the LCS in a coastal area. To accomplish it, we compute the FSLE from an ocean circulation model with realistic winds in two different situations: with and without gradients of the radiation stress tensor, to model the presence/absence of surface gravity waves. Finally, results are compared with available data from drifters.

\section{Data and Methods}

\subsection{Data}

The study has been performed in a semi-enclosed bay located in the southern side of the Island of Mallorca, Western Mediterranean Sea (Figure 1). Velocity data were obtained from the Regional Ocean Model System (ROMS), a free-surface, hydrostatic, 

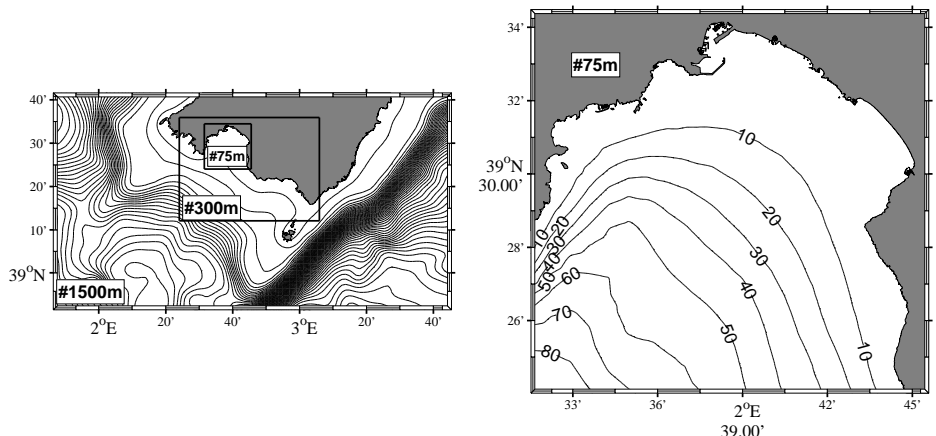

Fig. 1 Area covering the domains for the wave and current models (left). Fine resolution grid detail (right).

primitive equation ocean model that uses stretched, terrain-following coordinates in the vertical and orthogonal coordinates in the horizontal [20].

Three different domains were implemented in order to obtain high resolution currents in the area of study. The coarser mesh has a resolution of $d \theta=d \lambda=1^{\circ} / 74$ (e.g. $d x \simeq d y=1500 \mathrm{~m}$ ). This domain is nested to a second domain with a mesh of $d x=d y=300 \mathrm{~m}$, and the latter to a third domain covering the study area which has a grid resolution of $d x=d y=75 \mathrm{~m}$ with $348 \times 260$ nodes and 10 vertical levels (Figure 1 , right). This area is around $18 \mathrm{~km}$ wide with depths at its open boundary around 80 m. The Bay is open to southerly to south-westerly swells.

All domains were forced using winds provided by the PSU/NCAR mesoscale model MM5 [8]. The vertical structure of temperature and salinity was obtained from Levitus database $[1,14]$.

The study was done for conditions from November $10^{\text {th }}$ to November $24^{\text {th }} 2009$. During this period two different simulations were performed by forcing the ocean 
model with realistic wind fields (hereafter set I), and forcing the model with the same wind fields plus the additional gradients of the radiation stress tensor fields (set II).

For set II, the effects of waves are included in the three domains by adding the gradient of the radiation stresses which describe the excess of momentum flux caused by surface waves [15]. These terms have been added to the horizontal momentum equations at the free surface in order to properly represent the effect of the phase averaged waves in the mean flow [21]. The radiation stresses were obtained in the same domains of the circulation model by integrating WAM model, a third generation spectral wave model specifically designed for global and shelf sea applications [12].

Boundary conditions for the first domain were taken from the operational model for the Western Mediterranean operated by the Spanish Harbour Authority (Puertos del Estado). Although the wave model is not appropriate for very shallow waters since typical physical processes at those areas, such as diffraction, triad-wave interactions or depth-induced wave breaking are not considered, the model provides for typical wavelengths the correct wave field for depths higher than $10 \mathrm{~m}$. Stresses were updated into the ROMS model every three hours being internally interpolated between two consecutive values.

Additionally, on November $19^{\text {th }}$ eight drifting buoys were deployed in the area of study for three days. The buoys were specifically designed for coastal studies and they provide the position through GPS positioning transmitting via GSM every 5 minutes. 
2.2 Methods

From the velocity fields obtained in sets I and II we obtain the FSLE at an instant $t$ by computing the time, $\tau$, required for two fluid particles (one of them placed at point $\mathbf{x}$ ) to separate from the initial distance $\delta_{0}$ to the final one $\delta_{f}$,

$$
\lambda\left(\mathbf{x}, t, \delta_{0}, \delta_{f}\right)=\frac{1}{\tau} \ln \frac{\delta_{f}}{\delta_{0}}
$$

where $\delta_{0}$ coincides with the grid size for the velocity $(75 \mathrm{~m})$, and $\delta_{f}$ will be discussed in the next section in order to estimate the best value for our studies. Specifically, of the four grid neighbouring particles at distance $\delta_{0}$, we take the smallest of the times they take to reach $\delta_{f}$ (this minimum time is $\tau$ ). It is also important to note that we will mainly compute the FSLE evolving backwards-in-time the particle trajectories, since the LCS computed in this way are the ones with a direct physical interpretation [6]. However, to obtain the optimal $\delta_{f}$ we will first make an experiment computing them both, backwards and forward in time. Following the notation introduced by [6] we will represent FSLE computed backwards in time as $\lambda^{-}$whereas FSLE computed forward in time as $\lambda^{+}$.

\section{Results}

We have performed simulations with the circulation model for the considered period. The baroclinic time step for the model is set to $15 \mathrm{~s}$ and we store the surface velocity fields every 5 minutes. For both sets I and II the wind forcing and waves are interpolated every 5 minutes from the original 3 hour-resolution fields. Additionally, to compute the FSLE, four sets of virtual particles are launched in the third domain. 


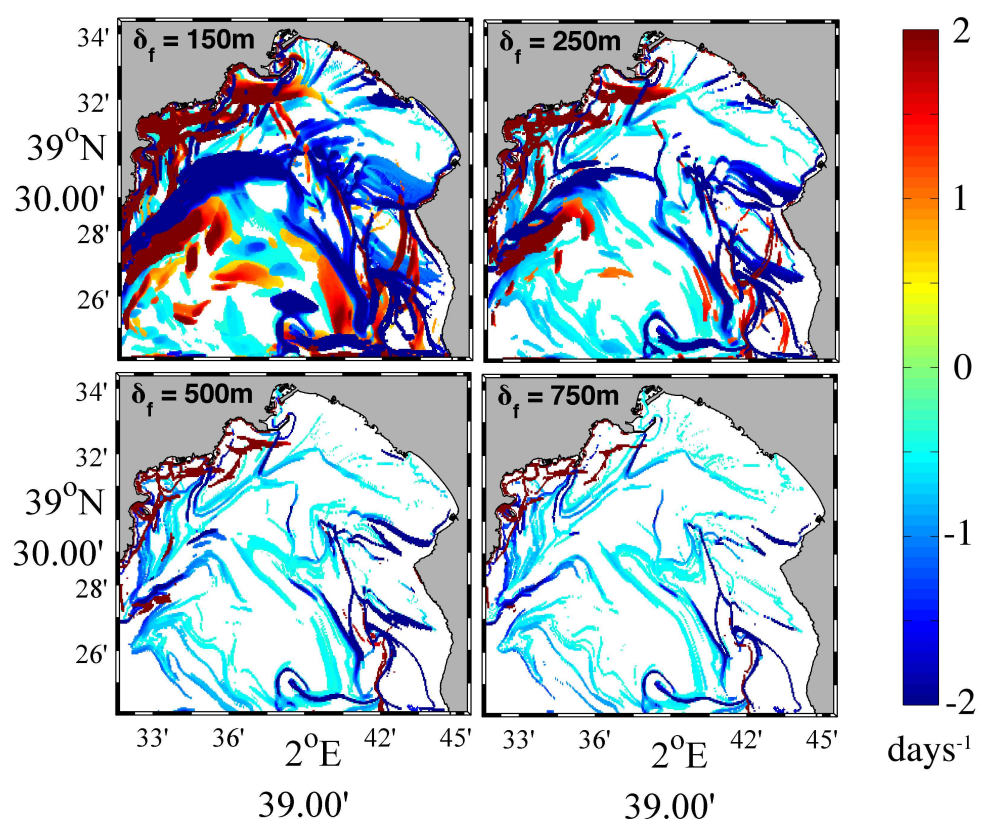

Fig. 2 FSLE for final separation of $\delta_{f}=150 \mathrm{~m}\left(\delta_{f} / \delta_{0} \approx 2\right), \delta_{f}=250 \mathrm{~m}\left(\delta_{f} / \delta_{0} \approx 3.3\right), \delta_{f}=500 \mathrm{~m}$ $\left(\delta_{f} / \delta_{0} \approx 6.7\right)$ and $\delta_{f}=750 \mathrm{~m}\left(\delta_{f} / \delta_{0} \approx 10\right)$ November $23^{\text {rd }}, 2009$ at $18: 00 \mathrm{~h}$. Just for plotting, we have considered positive the values of $\lambda^{+}$, and negative the ones for $\lambda^{-}$.

Particles were advected using a first order Euler algorithm which integrate in time the velocity data from the numerical model.

Firstly, we try to estimate the optimal $\delta_{f}$. For this we performed for a given day (November $23^{\text {rd }} 2009$ at 18.00 h) simulations with set I and different values of $\delta_{f}=$ $150 \mathrm{~m}, \delta_{f}=250 \mathrm{~m}, \delta_{f}=500 \mathrm{~m}$ and $\delta_{f}=750 \mathrm{~m}$ (see Figure 2) where the maximum values of $\lambda^{+}$and $\lambda^{-}$are displayed.

Their typical filamentous structures that approximate the LCS are already clear and well defined with $\delta_{f}=500 \mathrm{~m}$ (Figure 2,c) which correspond roughly with the separation between 6 mesh points. To reduce computational costs we take this value 
to proceed with further analysis. As already stated, in the following the LCS are computed only for the backwards in time dynamics $\left(\lambda^{-}\right)$.

The influence of waves in the stirring of the surface layer area is assessed by analysing the LCS during two periods, $15^{\text {th }}-18^{\text {th }}$ and $21^{\text {st }}-24^{\text {th }}$ November, with different wave conditions.

Starting on November $15^{\text {th }}$ relatively mild wave conditions were present in the area. For this period, we compute $\lambda^{-}$for both set I (Figure 3, left panel) and set II (Figure 3, right panel). LCS are shown for November $15^{\text {th }}$ to November $18^{\text {th }}$ at 00.00h. Not surprisingly the structure of the LCS displays a similar pattern for both sets of data. A barrier parallel to the coast appears and it is present during all the period separating two areas in the shallow zone. Shallow areas are characterized by stronger mixing as seen by the complex pattern of the LCS. Spatially averaged values of $\lambda^{-}$for currents are $0.09,0.13,0.29$ and 0.21 days $^{-1}$ for November $15^{\text {th }}, 16^{\text {th }}$, $17^{\text {th }}$ and $18^{\text {th }}$, respectively, corresponding to mixing times of approximately $21,14.6$, 6.54 , and 9 days.

For the same period these values including the coupling of waves and currents (set II) are $0.13,0.13,0.28$ and 0.23 days $^{-1}$ (mixing times of 14.6, 14.6, 6.77 and 8.24 , days).

The general performance of LCS in describing the flow is usually assessed by using virtual neutrally buoyant Lagrangian particles [19]. In Figure 3 we also represent the positions of 296 virtual particles at four different locations for November $15^{\text {th }}-18^{\text {th }}$ for the two sets. As expected, similar paths are followed by the particles 
deployed under current conditions (Figure 3, left), and the particles deployed under waves and currents (Figure 3, right) that are attracted in both cases on the LCS.

A different situation is presented during November $21^{\text {st }}$ to November $24^{\text {th }}$. During this period, significant wave heights $\left(H_{s}\right)$ measured at deep waters reached $1 \mathrm{~m}$. The effect of waves is to increase mixing and therefore to modify the transport at the surface. The LCS for this period are displayed each day at 00.00 in Figure 4 for set I (left) and for set II (right). Mean values of the wind stress imposed in set I during this period is $0.1 \mathrm{~N} / \mathrm{m}^{2}$. For set II, mean values of stresses (wind plus radiation from waves) increased up to $0.25 \mathrm{~N} / \mathrm{m}^{2}$. The barrier that was located near the coast during the analyzed mild wave conditions of November $15^{\text {th }}-18^{\text {th }}$ has now moved onshore appearing new areas of strong mixing in the middle of the Bay and new LCS are present for both set I and set II with larger values of $\lambda^{-}$in set II . Contrarily to the previous situation, the LCS from set I and the LCS from set II provide different patterns. In general, the effect of waves is to increase the number of LCS, or rather to make them more convoluted. The spatially averaged values of $\lambda^{-}$for set I are 0.35 , 0.45, 0.37 and 0.59 days $^{-1}$ (mixing times of 5.4, 4.2, 5.1 and 3.2, days) for currents at November $21^{\text {st }}, 22^{\text {nd }}, 23^{\text {rd }}$ and $24^{\text {th }}$ respectively. For the same days the spatially averaged values of $\lambda^{-}$for the coupling of waves and currents are respectively 0.41 , $0.49,0.42$ and 0.64 days $^{-1}$ (mixing times of 4.6, 3.9, 4.5 and 2.9, days). Again, we deployed 296 virtual particles in four different areas selected so as to be initially at different sides of any LCS. On November $22^{\text {nd }}$ the particles of set I launched in the south-eastern part of the Bay hardly moved, being the centroid located at the same position than 24 hours before. Contrarily, in set II, Lagrangian particles deployed on 


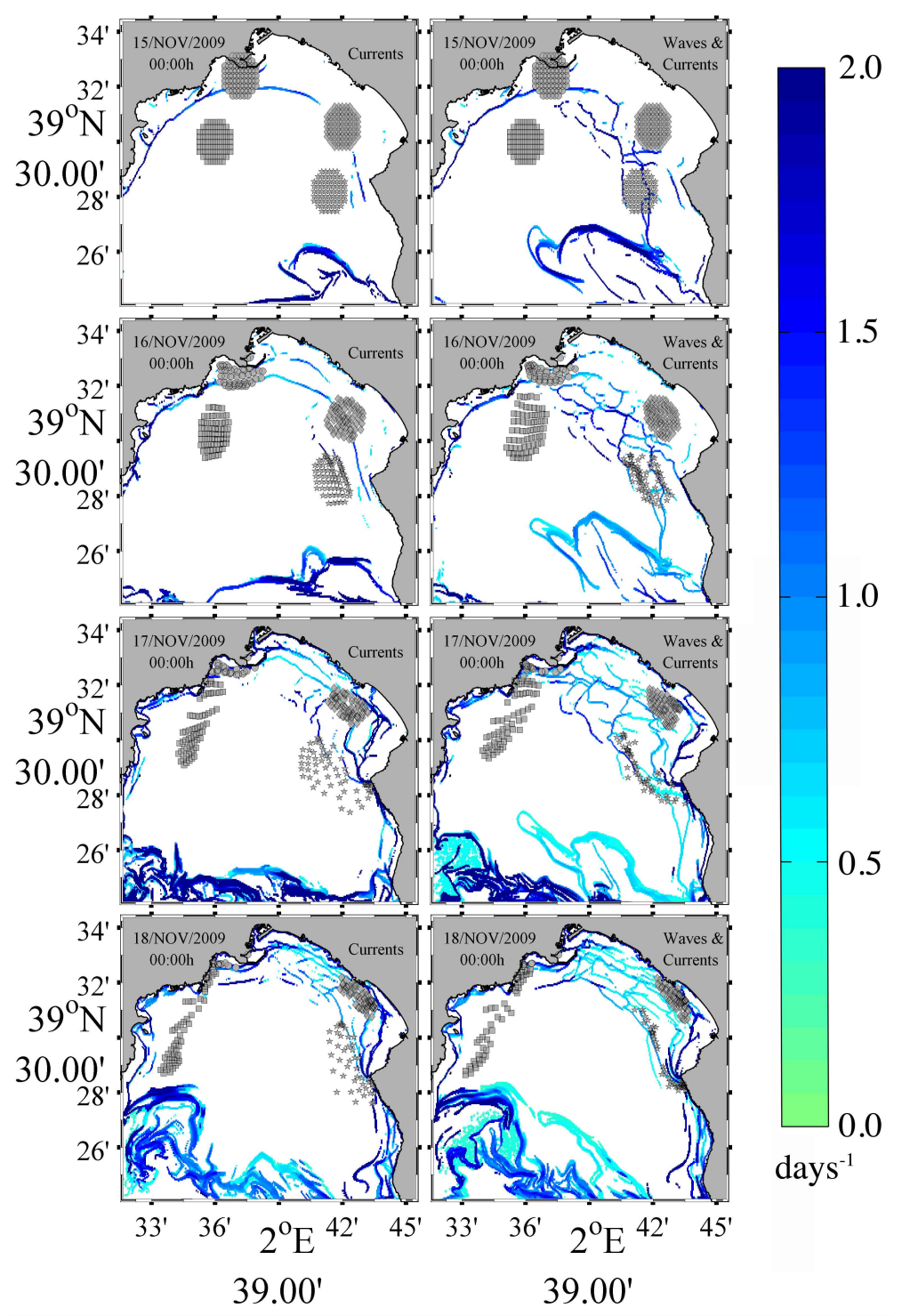

Fig. 3 Daily snapshots of $\lambda^{-}$, starting on November $15^{\text {th }}$ (mild wind conditions), computed for only currents (left panel), and with waves and currents (right panel). Virtual particles at four different areas are marked by circles, diamonds, squares and stars. 


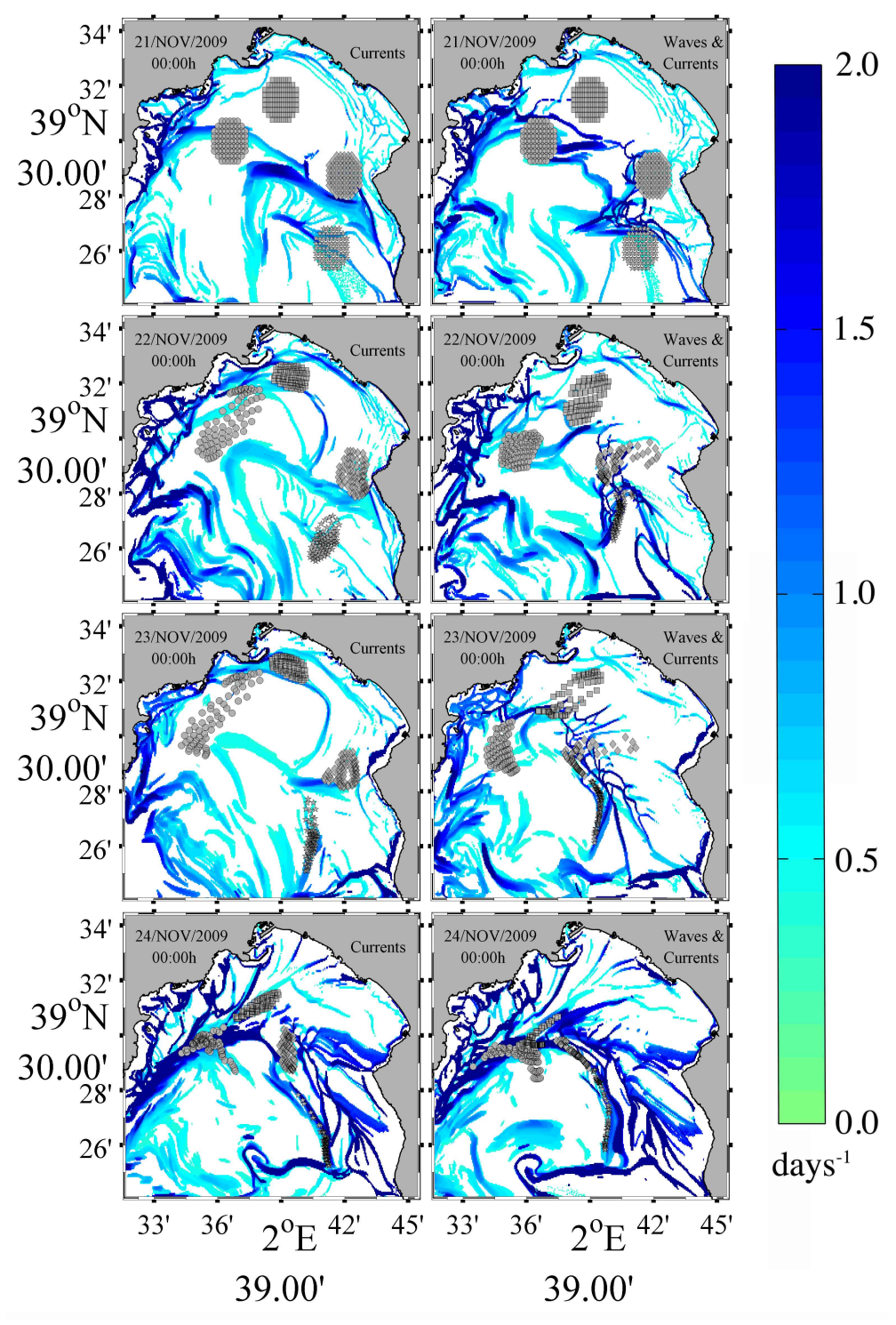

Fig. 4 Daily snapshots of $\lambda^{-}$, starting on November $21^{\text {st }}$ (strong wind conditions), computed by currents (left panel) and with waves and currents. Virtual particles at four different areas are marked by circles, diamonds, squares and stars. 
the eastern part of the Bay (diamonds and stars) are well mixed, and they start to allocate along the LCS that is being generated in the north-south direction (second row of Figure 4). One day later, on November $23^{\text {rd }}$, differences become more evident. The two groups of particles originally deployed on the eastern side, moved to the center of the Bay. The same snapshot for set I shows a very different situation, where the two groups evolve in a different way attracted by two weaker LCS. On the evening of November $23^{\text {rd }}$ wave heights decrease. Both LCS fields on November $24^{\text {th }}$ are very similar (Figure 4, bottom column). Obviously, due to the history of the dynamics, particles are disposed over different lines of attraction. Besides, particles deployed initially at different areas are totally mixed in set II (bottom row in Figure 4).

To further explore the role of the waves on the mixing in coastal areas coastal areas, four pairs of Lagrangian drifters were deployed on November $19^{\text {th }}$ at $12.00 \mathrm{~h}$. The position of the drifters are displayed, every 6 hours, together with the computed LCS for sets I and II in Figures 5 and 6 respectively. In these figures the trajectory of the buoys is also shown. During the first 18 hours $H_{s}$ was below $0.3 \mathrm{~m}$ and this is reflected in the pattern of the LCS. During this period, there is a clear separation between two areas of the Bay, with the deeper part more diffusive than the shallower one since currents for this conditions are mainly driven by the shelf slope dynamics. Drifters in the shallow part travel short distances indicating large residence times of surface waters. These buoys are located on a small diffusive zone for both set I (Figure 5) and set II (Figure 6). We want to recall at this point that since LCS are material surfaces particles can not travel across them. This fact is violated in set I where a drifter crosses the ridge of the $\lambda^{-}$that divides the Bay on the $20^{\text {th }}$ (second 
row of figure 5). More realistically, this LCS does not exist for set II (second row of figure 6), giving a natural pathway for the drifter. In the rest of the snapshots one also sees that the Lagrangian drifters follow more clearly the LCS from $\lambda^{-}$provided by set II. To quantify this fact, we compute the mean value of the FSLE in trajectories following by drifters in set I, which is near to 0.50 days $^{-1}$ while for set II, the same value is above 0.90 days $^{-1}$, i.e., drifters follow the LCS computed by set II in a better way than LCS computed in absence of waves.

\section{Conclusions}

We have shown that FSLE can be used, by choosing the proper scale, to analyze the dynamical field of coastal areas. We estimate that in coastal areas, a good representation of the Lagrangian dynamics is obtained by computing the FSLE with a final separation of Lagrangian particles of $\delta_{f}=500 \mathrm{~m}$. Additionally, a proper characterization of currents using coupled models of waves and currents is necessary to obtain the correct patterns of LCS which describe the dynamics of the area. The analyzed situation suggests that the effects of waves is to increase coastal mixing up to $10-20 \%$ with respect to the mixing induced by only-wind driven currents. The dynamical point of view provided by the analysis of the FSLE is a valuable tool to extract information about transport, mixing and residence time embedded in the Eulerian time dependent velocity fields obtained from numerical models or High Frequency Radar systems. 


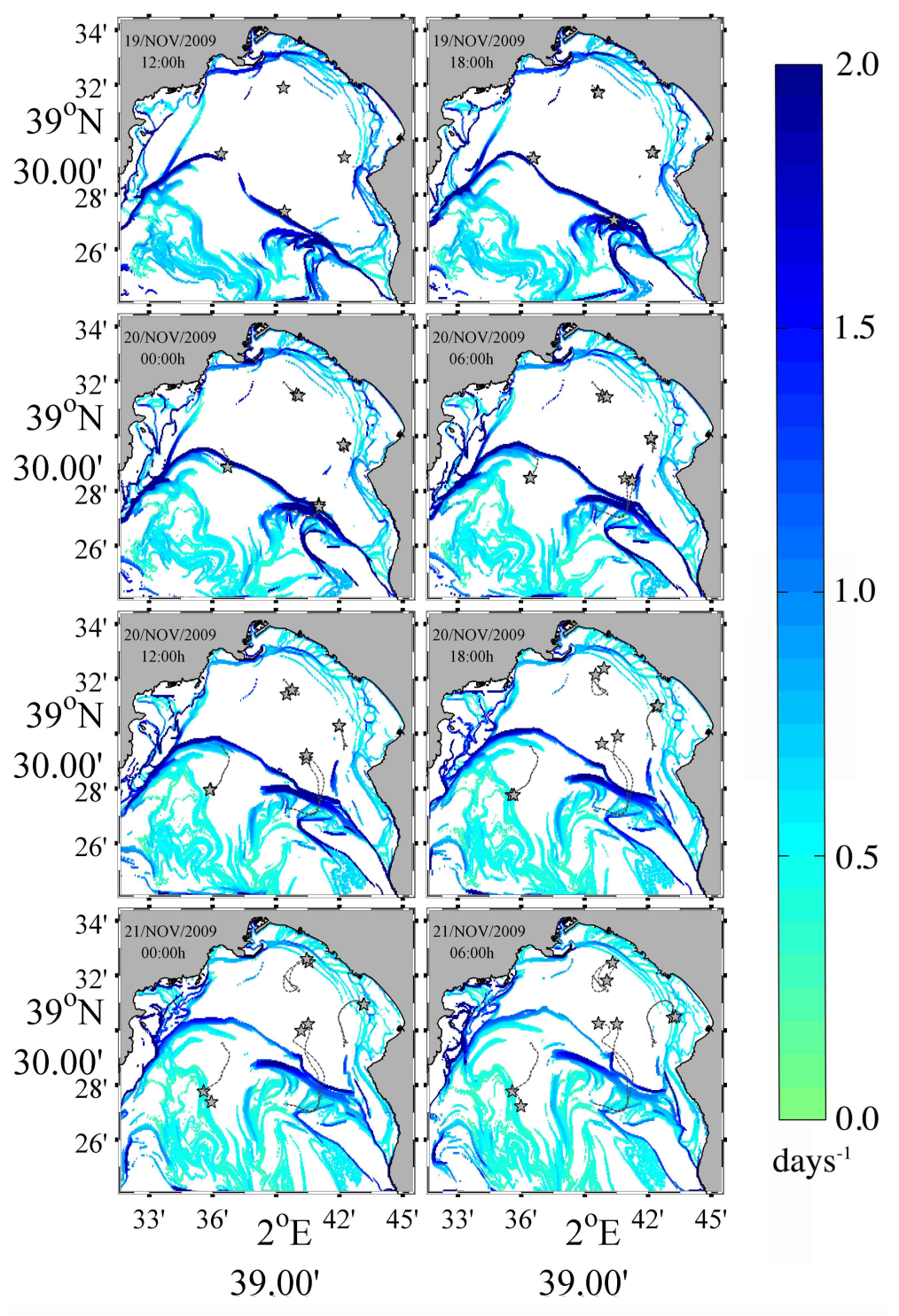

Fig. 56 hour snapshot of $\lambda^{-}$computed for only currents (set I), and trajectories of Lagrangian drifters.

\section{Acknowledgements}

Authors would like to thank financial support from Spanish MICINN thought projects

CTM2010-16915; CGL2011-22964 and from EU through MED Programme Project TOSCA (G-MED09-425). 


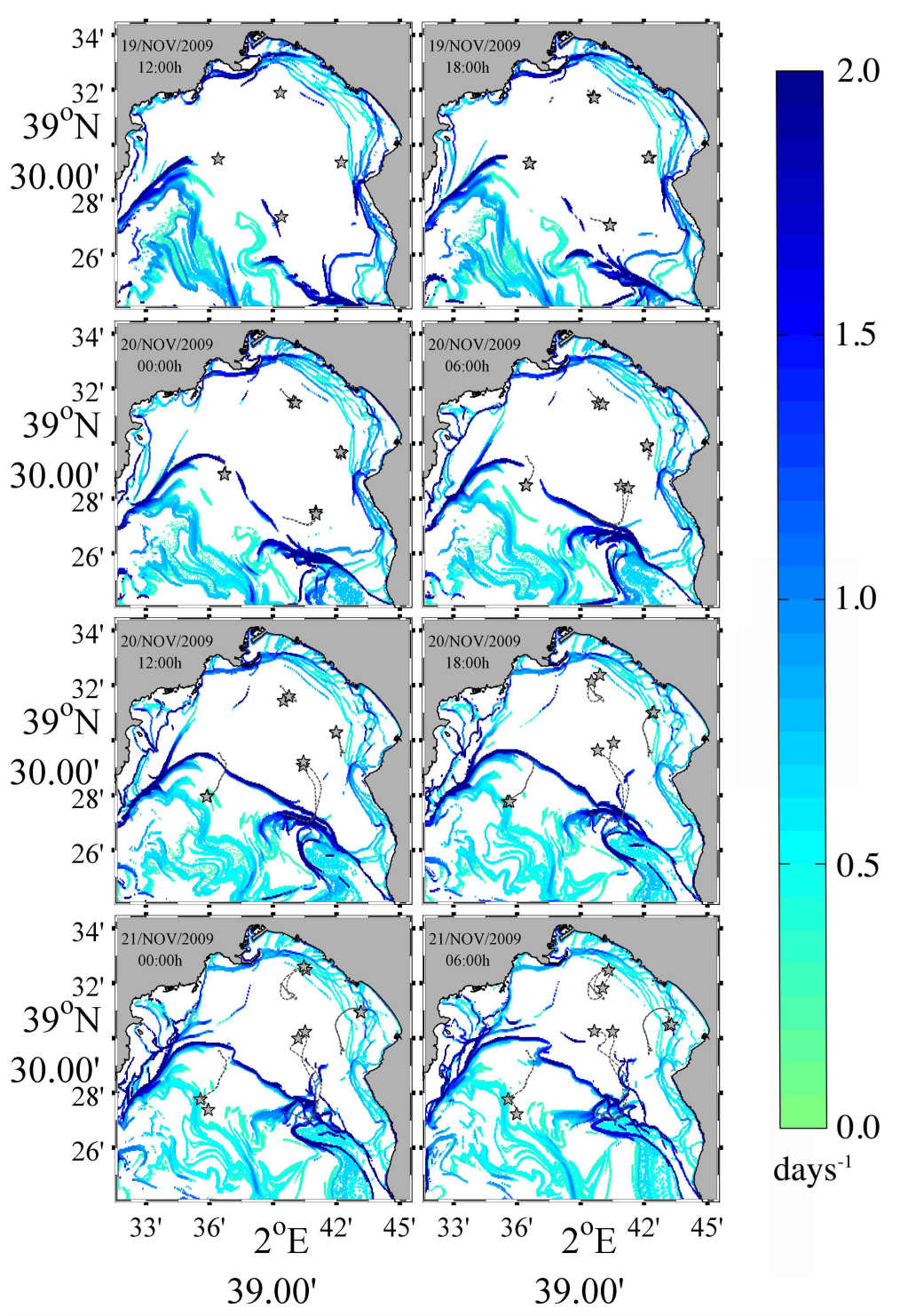

Fig. 66 hour snapshot of $\lambda^{-}$computed for currents and waves (set II), and trajectories of Lagrangian drifters.

\section{References}

1. Antonov, J. I., R. A. Locarnini, T. P. Boyer, A. V. Mishonov, and H. E. Garcia (2006), World Ocean Atlas 2005, Volume 2: Salinity. S. Levitus, Ed. NOAA Atlas NESDIS 62, U.S. Government Printing 
Office, Washington, D.C., $182 \mathrm{pp}$.

2. Artale, V., G. Boffetta, A. Celani, M. Cencini, and A. Vulpiani (1997), Dispersion of passive tracers in closed basins: Beyond the diffusion coefficient. Physics of Fluids, 9:3162-3171.

3. Aurell, E., G. Boffetta, A. Crisanti, G. Paladin, and A. Vulpiani (1997), Predictability in the large: an extension of the concept of Lyapunov exponent. Journal of Physics A 30:1-26.

4. Boffetta, G., G. Lacorata., G. Readelli, and A. Vulpiani (2001), Detecting barriers to transport: a review of different techniques. Physica $D, 159: 58-70$.

5. Buffoni, G., P. Falco, A. Griffa, and E. Zambianchi (1997), Dispersion processes and residence times in a semi-enclosed basin with recirculating gyres: An application to the Tyrrhenian sea. Journal of Geophysical Research - Oceans, 102:18699-18713

6. d’Ovidio, F., V. Fernández, E. Hernández-García and C. López (2004), Mixing structures in the Mediterranean sea from Finite-Size Lyapunov Exponents, Geophys. Res. Lett, 31, L17203, doi:10.1029/2004GL020328.

7. Gildor, H., E. Fredj, J. Steinbuck, and S. Monismith (2009), Evidence for submesoscale barriers to horixontal mixing in the ocean from current measurements and aerial photographs, J. Phys. Oceanogr. 39, 1975-1983.

8. Grell, G. A., J. Dudhia, and D. R. Stauffer (1995), A description of the fifth-generation Penn state/NCAR mesoscale model (MM5), NCAR/TN-398+STR, National Center for Atmospheric Research, Boulder, $C O, 122 \mathrm{pp}$.

9. Haller, G. and G. Yuan (2000), Lagrangian coherent structures and mixing in two-dimensional turbulence, Physica D, 147, 352-370.

10. Haza, A.C., T. M. Özgökmen, A. Griffa, A. Molcard and P. M. Poulain, Transport properties in small scale coastal flows: relative dispersion from VHF radar measurements and in the Gulf of La Spezia, Ocean Dynamics, (60), 4, 861-882, (2010).

11. Joseph, B. and B. Legras (2002), Relation between kinematic boundaries, stirring, and barriers for the Antarctic Polar vortex, Journal of the Atmospheric Sciences, 59, 1198-1212.

12. Komen, G.J., L. Cavaleri, M. Donelan, K. Hasselmann, S. Hasselmann and P.A.E.M. Janssen (1994), Dynamics and Modelling of Ocean Waves, Cambridge University Press

13. Lapeyre, G. (2002), Characterization of finite-time Lyapunov exponents and vectors in twodimensional turbulence, Chaos, 12 (3), 688-698, doi:10.1063/1.1499395. 
14. Locarnini, R. A., A. V. Mishonov, J. I. Antonov, T. P. Boyer, and H. E. Garcia (2006), World Ocean Atlas 2005, Volume 1: Temperature. S. Levitus, Ed. NOAA Atlas NESDIS 61, U.S. Government Printing Office, Washington, D.C., $182 \mathrm{pp}$.

15. Longuet-Higgins, M.S. and R.W. Stewart (1964), Radiation stresses in water waves: a physical discussion with applications, Deep Sea Res., 11, 529-562.

16. Molcard, A.,A.C. Poje, and T.M. Özgökmen (2006), Directed drifter launch strategies for Lagrangian data assimilation using hyperbolic trajectories, Ocean Modelling, 12, 268-289.

17. Özgökmen, T.M., A. Griffa, A.J. Mariano and L.I. Piterbarg (2000), On the predictability of Lagrangian trajectories in the ocean, J. Atmos. Ocean. Tech., 17 (3), 366-383.

18. Shadden, S. C., F. Lekien and J. E. Marsden (2005), Definition and properties of Lagrangian coherent structures from finite-time Lyapunov exponents in two-dimensional aperiodic flows, Physica D, 212, (3-4), 271-304.

19. Shadden, S.C., F. Lekien, J. D. Paduan, F. P. Chavez, and J. E. Marsden (2009), The correlation between surface drifters and coherent structures based on high-frequency radar data in Monterey Bay, Deep Sea Research Part II: Topical Studies in Oceanography 56, 161-172.

20. Song, Y. and D. B. Haidvogel (1994), A semi-implicit ocean circulation model using a generalized topography-following coordinate system, J. Comp. Phys., 115 (1), 228-244.

21. Warner, J.C., Sherwood, C.R., Signell, R.P., Harris, C.K. and Arango, H.G. (2008), Development of a three-dimensional, regional, coupled wave, current, and sediment-transport model, Computers \& Geosciences, 34 (10), 1284-1306 